\title{
Photometric and Polarimetric Interpretation of Blazar AO 0235+164 Behaviour
}

\author{
Elena Shablovinskaya \\ Department of Astrophysics, Faculty of Mathematics and Mechanics, Saint Petersburg State University, \\ Saint Petersburg 199034, Russia; gaerlind09@gmail.com \\ Academic Editors: Milan S. Dimitrijević and Luka Č. Popović \\ Received: 7 September 2017; Accepted: 3 October 2017; Published: 19 October 2017
}

\begin{abstract}
Among the great number of controversial issues, the most topical one both for theoretical and observational astrophysics presently is a problem of active galactic nuclei investigation. To explain the behaviour of blazar AO 0235+164, which has been under observation at the LX200 telescope (SPbU) since 2002, the method of analyzing developed by V.A. Hagen-Thorn and S.G. Marchenko was applied. It is based on the assumption that in the case of observational data lying on the straight line in the absolute Stokes parameters space $\{I, Q, U\}$ (for polarimetry) and the fluxes space $\left\{F_{1}, \ldots F_{n}\right\}$ (for photometry), relative Stokes parameters and relative flux ratios stay unchanged, and consequently, only one source is corresponding to the variability of general value of flux. In this paper, the photometric and polarimetric interpretation of blazar behaviour is presented. Furthermore, the flux and flux-flux diagrams are obtained for three periods of object monitoring: 2006-2007 and 2008-2009 (outbursts) and 2009-2016 (decline with 2015 outburst).
\end{abstract}

Keywords: active galaxies; BL Lacertae objects; $\mathrm{AO} 0235+164$; photometry; polarimetry

\section{Introduction}

One of the leading problems of both theoretical and observational astrophysics today is the determination and investigation of the nature of extragalactic objects' activity (active galactic nuclei, AGN) . Currently, particular attention is paid to BL Lac objects considered to be compact active nuclei with a relativistic plasma jet closely aligned to the line of sight that provides non-thermal emission [1]. This type of orientation is able to clarify extremal properties of the sources such as violent variability and high degree of polarization.

One of the most intensively studied BL Lac objects is AO $0235+164$. It was classified as an AGN in 1975 [2]; in the same work, the variability in the optical and radio wavebands was detected. Furthermore, the object reveals different values of redshift: $z=0.940$ for emission light and $z=0.851$ and $z=0.524$ for absorption components, and for this reason there has been the conjecture of a gravitational microlensing mechanism [3]. Concerning the modern studies, the optical and radio light curves were analyzed, and the periodicity of the outburst on a $\sim 5.7 \mathrm{yr}$ time scale was suggested [4]. Moreover, intraday flux variability was also obtained at radio [5] and optical [6] wavebands and at high energies [7], while only at $\mathrm{cm}$-wavelength could it be explained with interstellar scintillation.

The attention of this paper is focused on the blazar AO 0235+164. The monitoring of the blazar has been carried out on the telescopes of Saint Petersburg State University 16-inch Schmidt-Cassegrain reflector LX200 (Peterhof, Saint Petersburg) and 27.6-inch Cassegrain reflector AZT-8 (Crimean Astrophysical Observatory) since 2002. The long observational data set will be given and analyzed; the source properties will be revealed as well. 


\section{Analysis Method}

Modern research is concerned with the estimation of active galaxies-and BL Lacertae objects particularly-as complicated systems where the underlying or "host" galaxy, inner emission region (accretion disk, broad line region (BLR), central engine), different parts of jet, etc. make contributions to the total flux. It is also considered that the source responsible for variations is located in the area close to the central engine. As active galaxies cannot yet be resolved into separated components by means of direct observations, the source properties giving an idea of the nature of nuclei activity can be revealed solely with indirect methods.

One of the essential source characteristics is the spectral energy distribution (SED). It can be obtained by gaining multicolour data on photometric variability. In that case, a long data set needs to be collected to judge the phase of object activity. Moreover, the most recognizable features of BL Lac objects' emission are high polarization degree (up to $60-70 \%$ at outbursts) [8] and variability of both polarization degree and angle.

To analyze the variable source behaviour, the method worked out by V. Hagen-Thorn and S. Marchenko was chosen. At its foundation lies the assumption that variability is caused by only one source. In order for the variations to imply only the total flux value changing while the relative Stokes parameters (for polarimetry) and relative SED (for photometry) stay unchanging, then in the space of absolute Stokes parameters $\{I, Q, U\}$ or in flux space $\left\{F_{1}, . . F_{n}\right\}$, the observational data should lie on a straight line (Figure 1). Moreover, the inclination angles are relevant to relative Stokes parameters and relative flux ratios in the chosen wavebands.
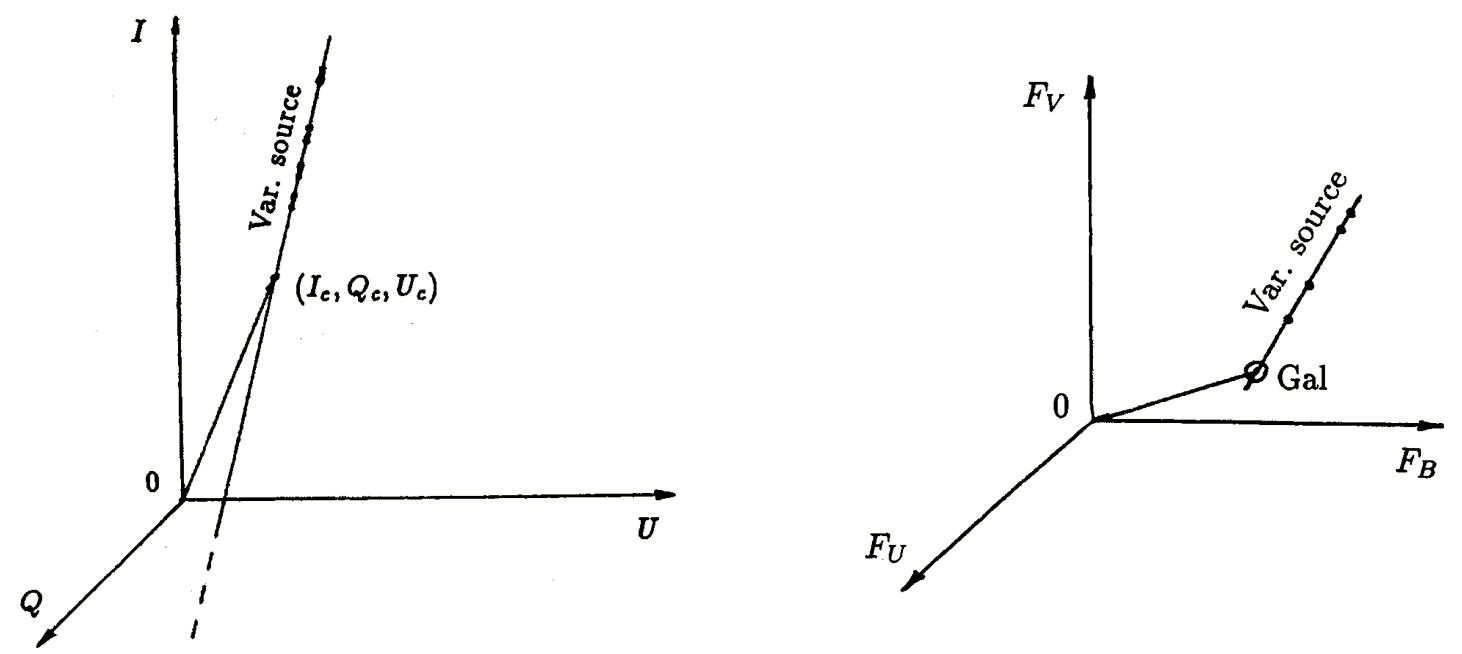

Figure 1. Polarization behaviour in space of absolute Stokes parameters (left) and photometry behaviour in three-dimensional flux space (right).

The method's application to the observational data requires the solution of the inverse task. As it was proven in [9], the opposite is also true: the linear relation of fluxes points at unchanging SED. Therefore, in the case of observational data lying on a straight line in the given space, only one source is corresponding to AGN variability. More details can be found at $[9,10]$.

\section{Observational Data}

The observational data was obtained at the telescopes of Saint Petersburg State University stations by CCD cameras ST-7XME (produced by SBIG). The CCD camera properties are reported in the Table 1 .

Photometric data was gained by multicolour observations with $B, V$ Jonhson and $R_{c}, I_{c}$ Cousins filters; calculated accuracy was $\approx 0.03^{m}$ [11]. Polarimetric data was provided with Savart plates rotated 
through $45^{\circ}$ with respect to each other. Observations were made at $R_{\mathcal{c}}$ waveband (AZT-8) and at the broad band with the effective wavelength close to $R_{c}$ filter (LX200). The error value was less then $2 \%$.

Table 1. Characteristics of the ST-7XME CCD camera.

\begin{tabular}{cc}
\hline Pixel number & $765 \times 510$ \\
Pixel size & $9 \times 9 \mu \mathrm{m}$ \\
Linear size & $6.9 \times 4.6 \mathrm{~mm}$ \\
Dark current & $1 \mathrm{e}^{-} / \mathrm{pixel} / \mathrm{sec}$ at $0{ }^{\circ} \mathrm{C}$ \\
RON & $15 \mathrm{e}^{-}$ \\
\hline
\end{tabular}

\subsection{Photometry}

Following [12] we could define three or more dimensional flux spaces, where flux-flux diagrams are projections to the relevant planes. Due to additivity, the flux can be presented as a sum of variable term $F^{v a r}$ and constant term $F^{c}$. In the case of a single source assumption with unchanging SED, flux ratios can be given as:

$$
F_{i}^{v a r} / F_{j}^{v a r}=c_{i j}
$$

where $c_{i j}=$ const . Comparing different wavebands it can be revealed that:

$$
F_{j}=c_{i j} F_{i}+\left(F_{j}^{c}-c_{i j} F_{i}^{c}\right),
$$

where $F_{j}$ and $F_{i}$ are observed fluxes. It is easily seen that the multicolour observations represented in this paper with four different wavebands can be used to divide the host galaxy and the variable source fluxes. Therefore, computing the inclination angles for the flux-flux dependencies, the flux ratios could be found, and according to them the supposition of colour changes or colour permanency could be done that gives an opportunity to consider the source behaviour.

For the blazar AO 0235+164, photometric data covering the period from 2006 to 2016 in filters $B$, $V, R_{c}, I_{\mathcal{C}}$ was analyzed. The light curves are represented in Figure 2. In order to convert magnitudes to fluxes, the conversion "magnitudes to fluxes" for given filters and CCD camera are presented in the Table 2 [11].

Thereby, the flux diagrams were plotted (Figure 3). It could be clearly seen that throughout the chosen period, two outbursts (violent flux increase ) took place-in 2006-2007 and 2008-2009, when the magnitude was up to $13^{m}$ (Figure 2), and also the period of decline-2009-2016, presumably, with the outburst in 2015. All of the data were divided to these three periods and flux-flux diagrams were studied as projections on planes in flux space $\left\{F_{B}, F_{V}, F_{R}, F_{I}\right\}$. The same periods were used for polarimetry interpretation. The data is presented in Figures 4-6.

Table 2. Calibration formulae for filters $B, V, R_{c}, I_{c}$.

\begin{tabular}{cc}
\hline Band & Logarithm of Flux $\boldsymbol{F}(\boldsymbol{v})(\mathbf{m J y})$ \\
\hline$B$ & $\lg F_{B}=6.629-0.4 B$ \\
$V$ & $\lg F_{V}=6.561-0.4 \mathrm{~V}$ \\
$R_{c}$ & $\lg F_{R}=6.489-0.4 R_{c}$ \\
$I_{C}$ & $\lg F_{I}=6.406-0.4 I_{C}$ \\
\hline
\end{tabular}


Atoms 2017, 5, 39

4 of 10
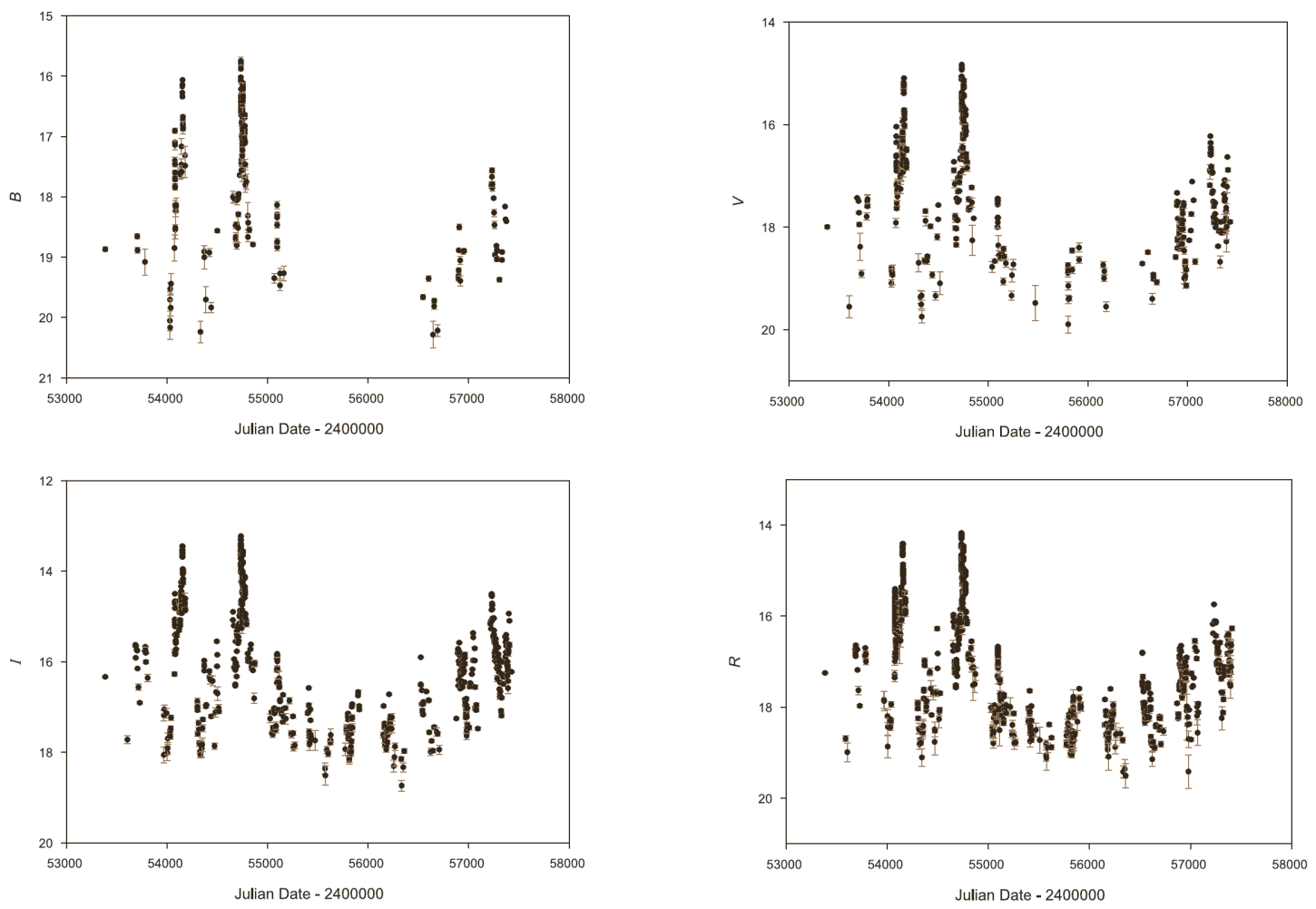

Figure 2. Light curves in filters $B, V, R_{c}, I_{c}$ for 2006-2016.
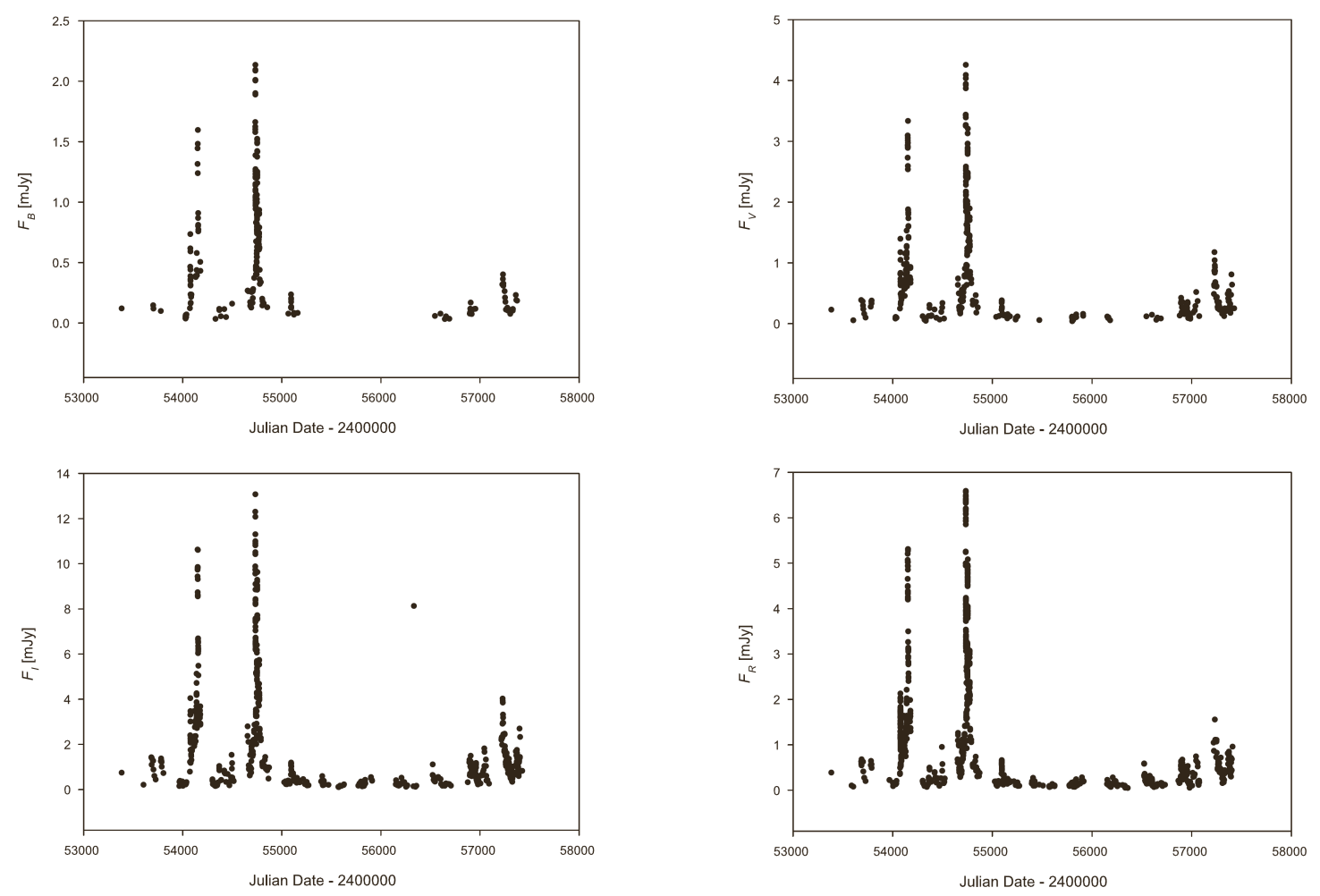

Figure 3. Flux diagrams in filters $B, V, R_{C}, I_{C}$ for 2006-2016. 

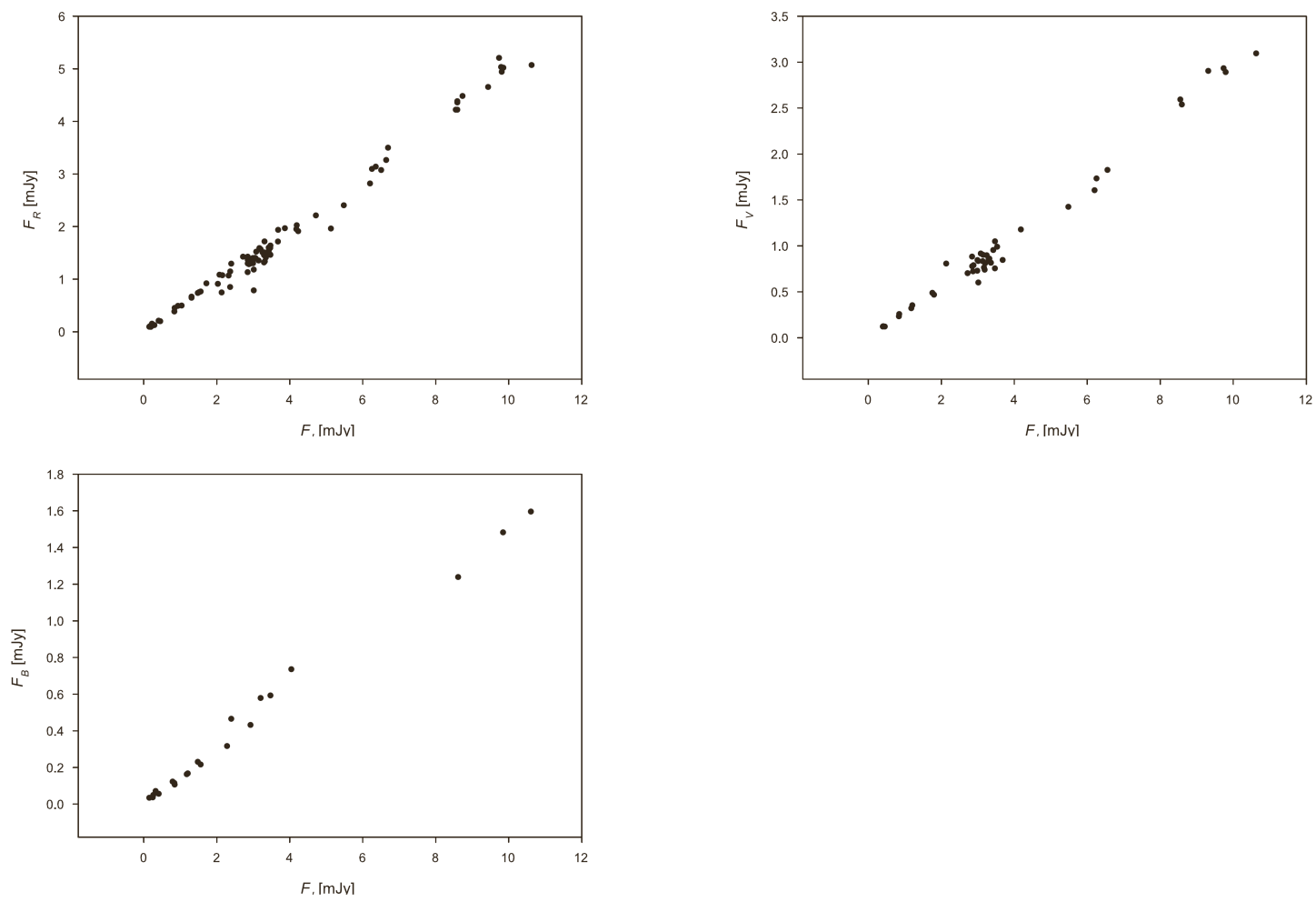

Figure 4. Flux-flux diagrams for outburst 2006-2007.
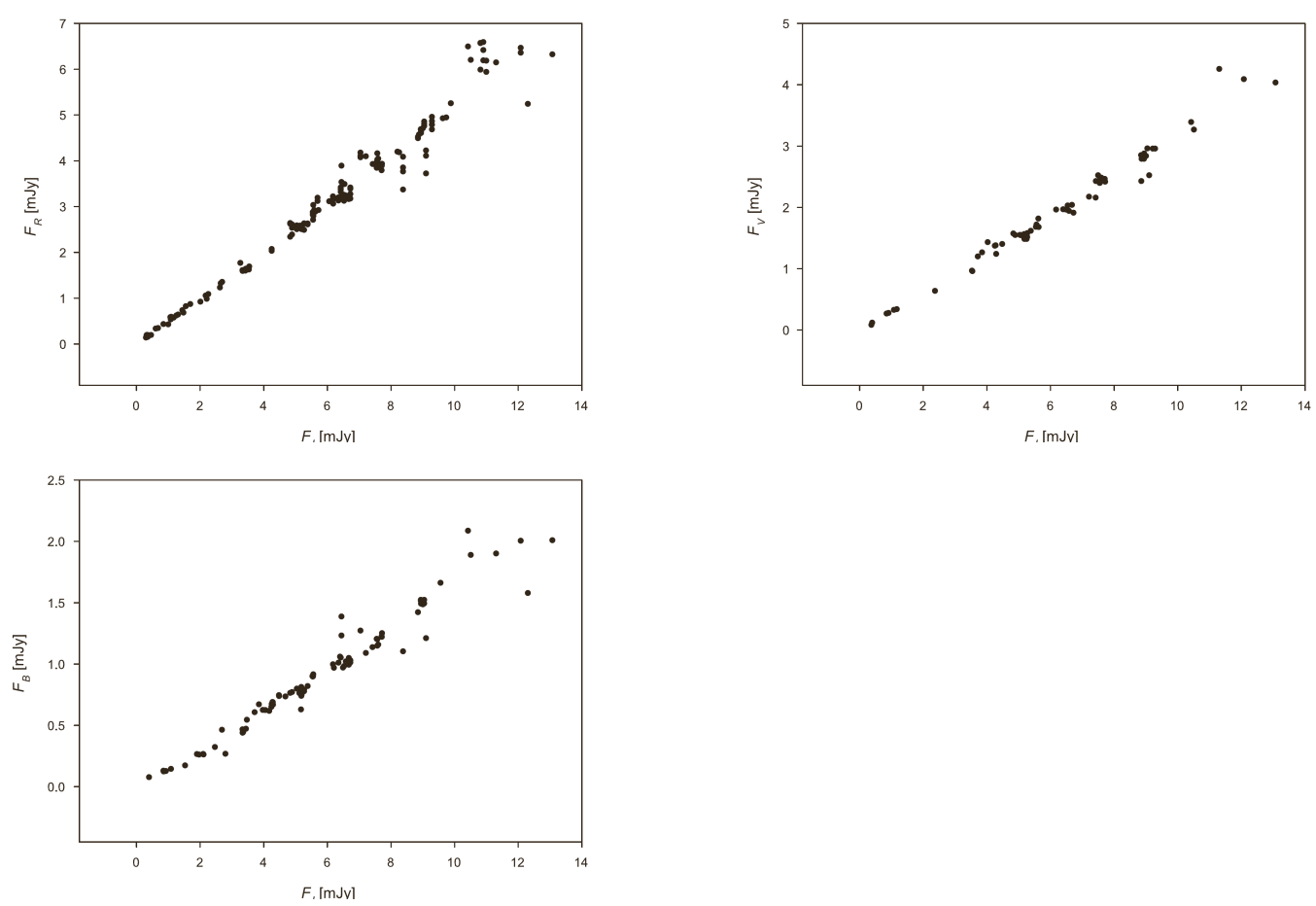

Figure 5. Flux-flux diagrams for outburst 2008-2009. 

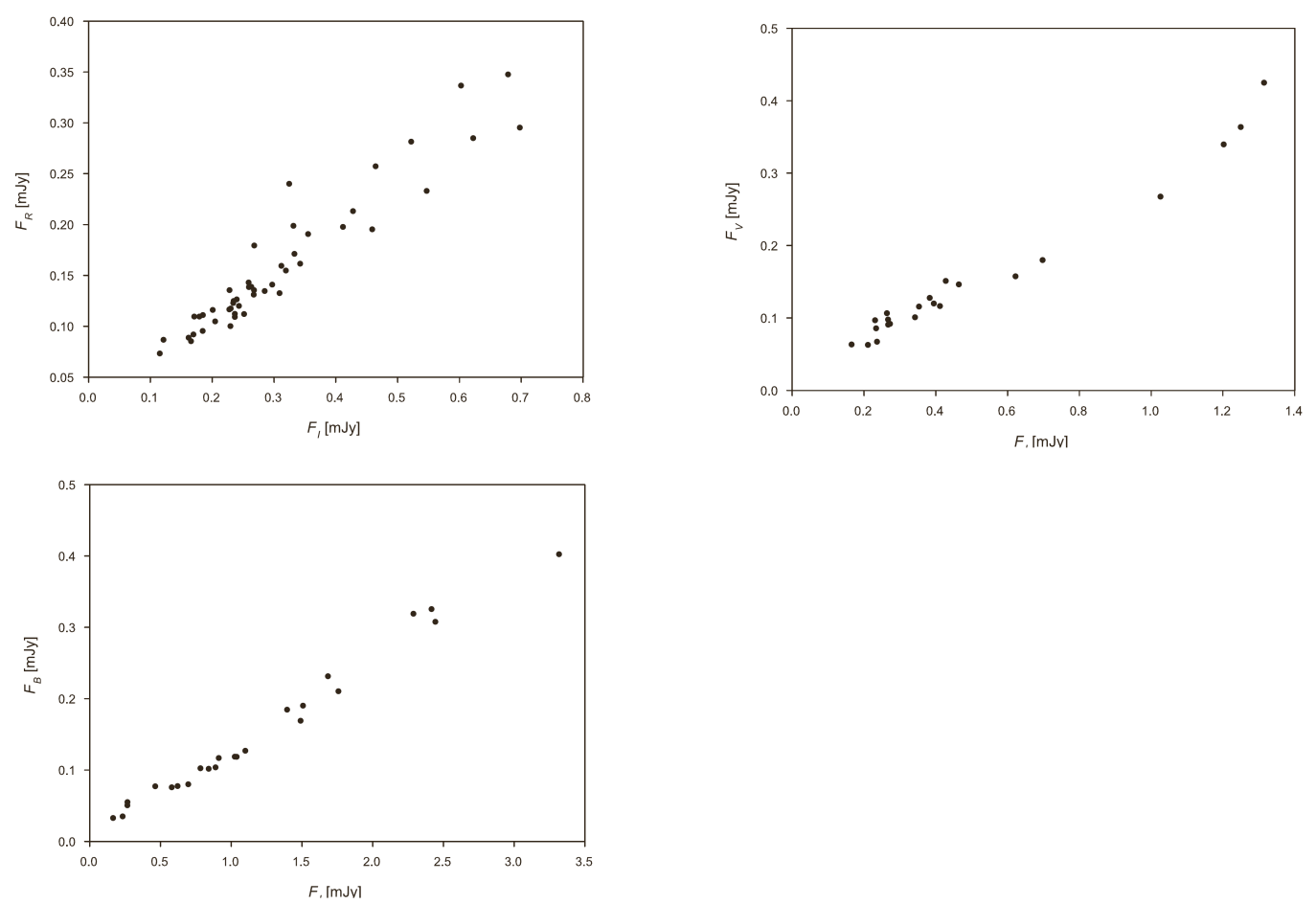

Figure 6. Flux-flux diagrams for decline 2009-2016.

\subsection{Polarimetry}

Polarimetric observations at the SPbSU station are dedicated to investigating the linear polarization - the $Q$ and $U$ Stokes parameters. The $I$ parameter is considered to be equal to the $F_{R}$ flux value. For data analysis, the same assumption and processing methods were used. As the Stokes parameters and photometric fluxes are additive, the observed values could be divided to the constant and variable parts: $Q^{c}, U^{c}$, and $I^{c}$, and $Q^{\text {var }}, U^{\text {var }}$ and $I^{\text {var }}$. Then, the relative Stokes parameters $p_{x}, p_{y}$ could be introduced:

$$
p_{x}=p \cos 2 \theta, p_{y}=p \sin 2 \theta,
$$

where $p$ is a polarization degree: $p=\left(p_{x}^{2}+p_{y}^{2}\right)^{1 / 2}, \theta$ is a polarization angle. $p_{x}, p_{y}$ are considered to be constant. The observed Stokes parameters could be presented as:

$$
\left\{\begin{array}{l}
Q=p_{x} I+\left(Q^{c}-p_{x} I^{c}\right) \\
U=p_{y} I+\left(U^{c}-p_{y} I^{c}\right)
\end{array}\right.
$$

The system apparently describes the equation of a straight line, where $p_{x}, p_{y}$ are direction tangents. To investigate the polarimetric behaviour in the case of the chosen method, the projections $Q(I)$ and $U(I)$ were plotted and studied for each period. The data is shown in Figures 7-10. 

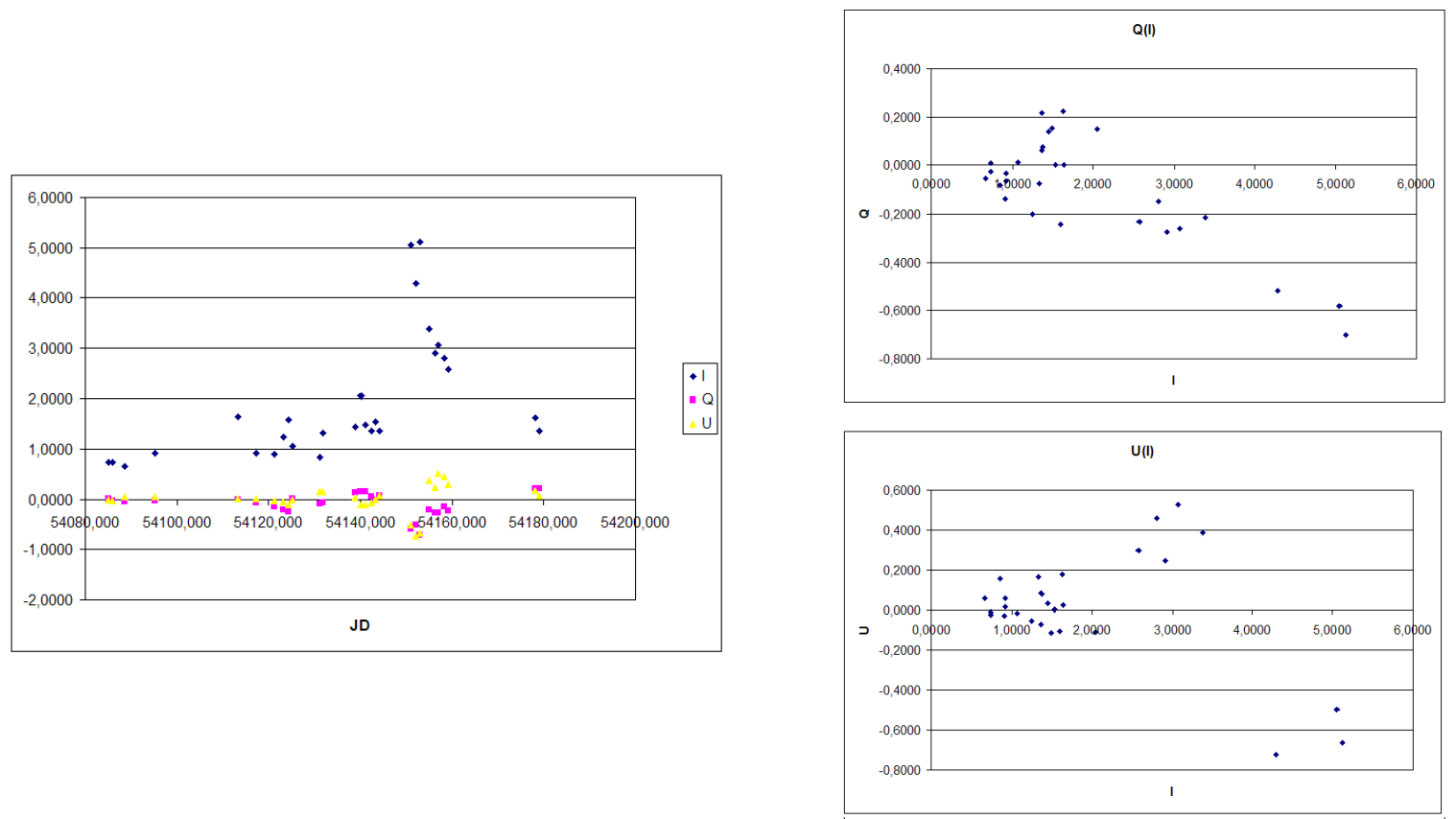

Figure 7. Absolute Stokes parameters behaviour (left) and $Q(I)$ and $U(I)$ (right) at the first period.
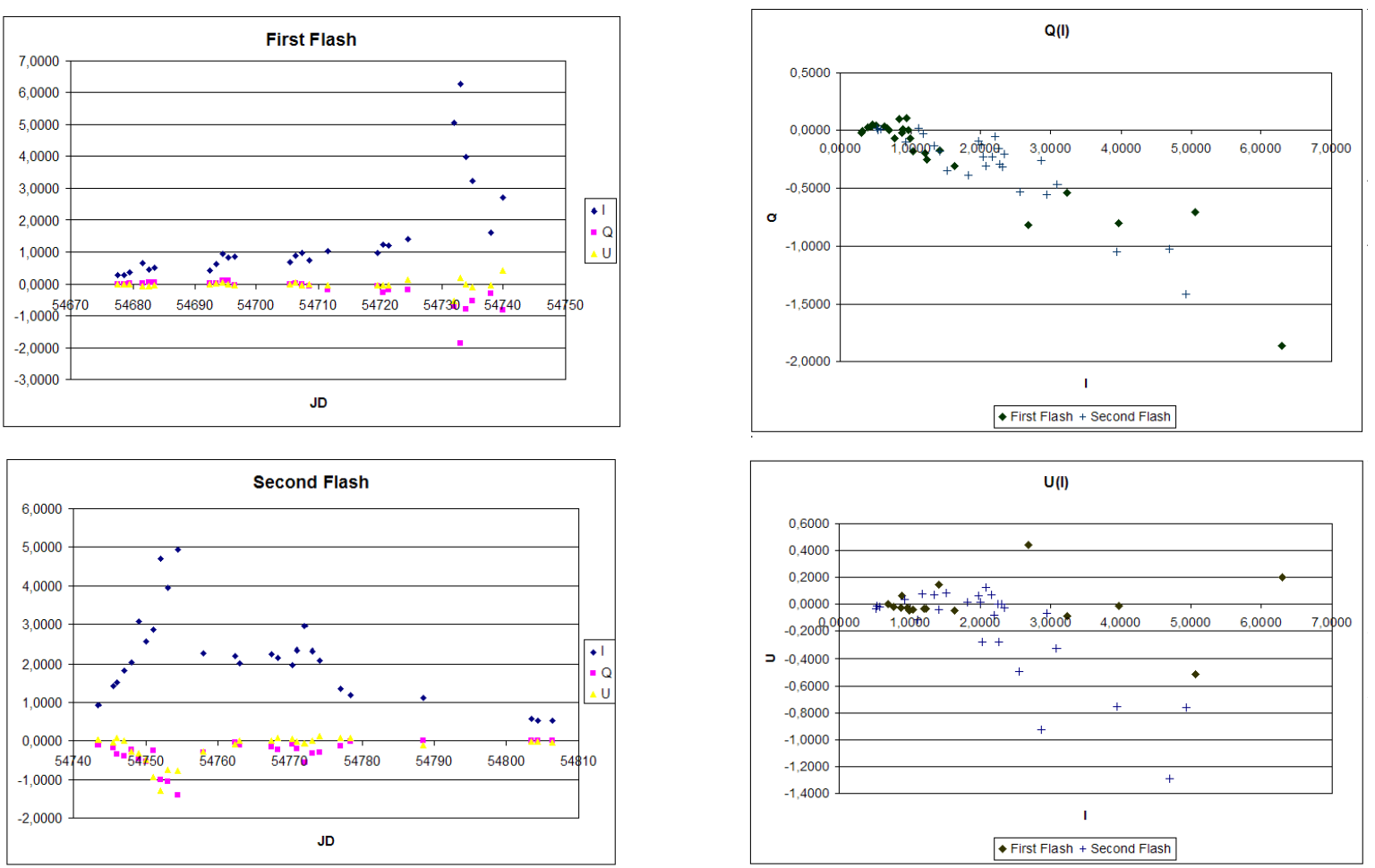

Figure 8. Absolute Stokes parameters behaviour at different flashes (left) and $Q(I)$ and $U(I)$ (right) at the second period. 

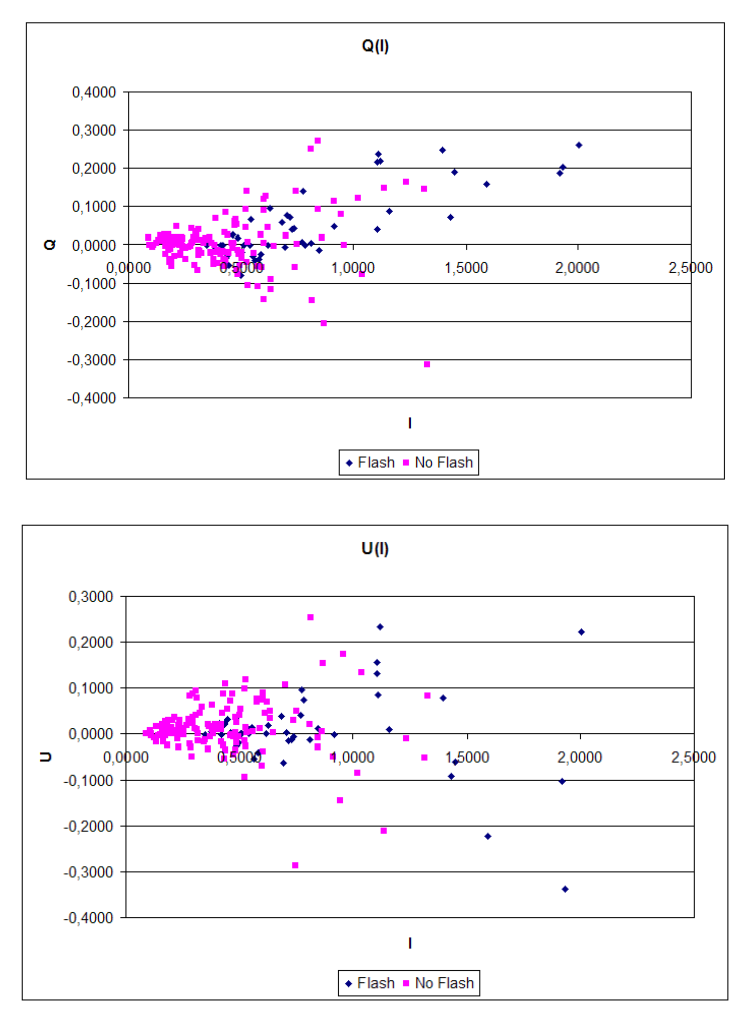
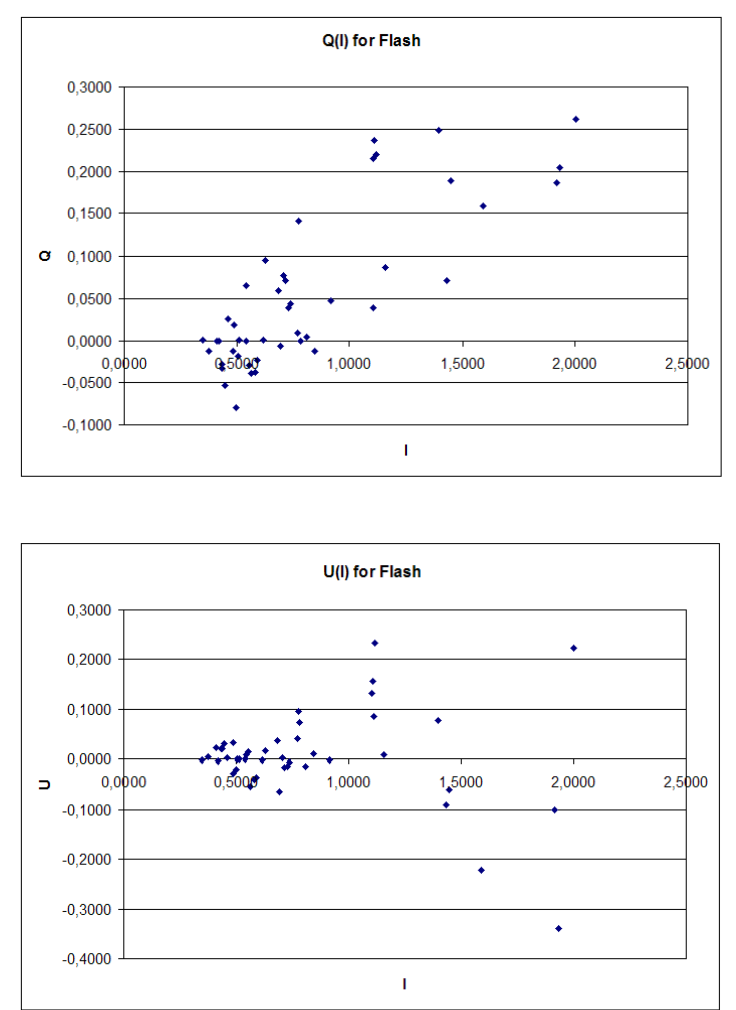

Figure 9. $Q(I)$ and $U(I)$ (left) and $Q(I)$ and $U(I)$ for the flash (right) at the third period.

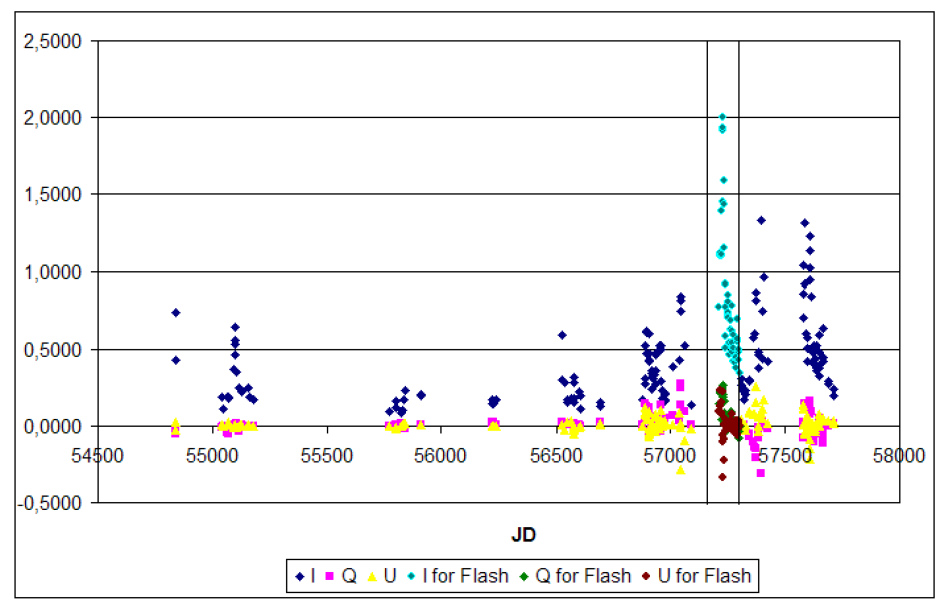

Figure 10. Absolute Stokes parameters behaviour at the third period (the flash is marked).

\section{Results}

Within the confines of the method, the obtained flux-flux curves could be approximated with a linear equation of type: $X=\alpha Y+\beta$, where $X$ and $Y$ are fluxes $F_{i}$ and $F_{j}$ or the Stokes parameters. As values of $X$ and $Y$ are loaded with errors, they are considered as random values distributed equally and not correlated. Thus, the method of orthogonal regressions of A. Wald described in [13] was applied. To calculate the inclination angle $\alpha$, the next expression was used:

$$
\operatorname{tg} 2 \alpha=2 \frac{\sum_{k=1}^{n}\left(x_{k}-\bar{x}\right)\left(y_{k}-\bar{y}\right)}{\sum_{k=1}^{n}\left(x_{k}-\bar{x}\right)^{2}-\sum_{k=1}^{n}\left(y_{k}-\bar{y}\right)^{2}}
$$


where $x_{k}$ and $y_{k}$ are values of $X$ and $Y$, respectively, and $\bar{x}$ and $\bar{y}$ are average values. Therefore, the inclination angles were calculated by photometric diagrams $F_{B}\left(F_{I}\right), F_{V}\left(F_{I}\right)$, and $F_{R}\left(F_{I}\right)$ (see Table 3) and by polarimetric diagrams $Q(I)$ and $U(I)$ (see Table 4) for three periods of the blazar activity.

Table 3. The inclination angles for photometric data.

\begin{tabular}{cccc}
\hline & $\boldsymbol{B}\left(\boldsymbol{I}_{\boldsymbol{c}}\right)$ & $\boldsymbol{V}\left(\boldsymbol{I}_{\boldsymbol{c}}\right)$ & $\boldsymbol{R}\left(\boldsymbol{I}_{\boldsymbol{c}}\right)$ \\
& $\boldsymbol{\delta} \boldsymbol{B}\left(\boldsymbol{I}_{\boldsymbol{c}}\right)$ & $\boldsymbol{\delta} \boldsymbol{V}\left(\boldsymbol{I}_{\boldsymbol{c}}\right)$ & $\boldsymbol{\delta} \boldsymbol{R}\left(\boldsymbol{I}_{\boldsymbol{c}}\right)$ \\
\hline Outburst & 0.151 & 0.280 & 0.455 \\
2006-2007 & \pm 0.034 & \pm 0.053 & \pm 0.029 \\
\hline Outburst & 0.162 & 0.308 & 0.485 \\
2008-2009 & \pm 0.007 & \pm 0.043 & \pm 0.006 \\
\hline Decline & 0.126 & 0.287 & 0.455 \\
2009-2016 & \pm 0.021 & \pm 0.005 & \pm 0.053 \\
\hline
\end{tabular}

Table 4. The inclination angles for polarimetric data.

\begin{tabular}{ccc}
\hline & $Q(I)$ & $U(I)$ \\
& $\delta Q(I)$ & $\delta U(I)$ \\
\hline Outburst & -0.156 & -0.130 \\
$2006-2007$ & \pm 0.013 & \pm 0.026 \\
\hline Outburst & -0.284 & -0.052 \\
2008-2009 & \pm 0.011 & \pm 0.011 \\
\hline Decline & 0.092 & -0.008 \\
2009-2016 & \pm 0.014 & \pm 0.014 \\
\hline
\end{tabular}

\section{Discussion}

From the presented flux-flux diagrams and polarimetric dependencies it is obvious that Choloniewski's method, extended by Hagen-Thorn and described in Section 2, could be applied to the interpretation of the behaviour of blazar AO $0235+164$. The possibility of the good photometric diagrams' approximation by straight lines reveals that within the two separated outbursts-2006-2007 and 2008-2009_fluxes ratios stayed unchanging, the object does not evince colour variability, and consequently, SED was permanent as well.

Furthermore, the comparison between the inclination angles at the different periods gives an approximate equality of all dependencies (the same filters are meaning) with the given accuracy. This could be mentioned as an indirect indication of the fact that physical properties of variable source were constant throughout the given event. In spite of possible multicomponent structure of radiating center, we could assume that the main contribution to the emission was made by a single source with a lifetime not less than the event duration. However, the data of decline period 2009-2016 noticeably differs from outbursts. It could be explained, firstly, by the lack of observations due to technical difficulties, and secondly, by gaining low fluxes with greater errors (and therefore less-accurate approximation). It could also be supposed that at the decline period the intensity of the variable source falls and the multicomponent structure displays changing SED.

According to the theory, the inclination angles of $Q(I)$ and $U(I)$ dependencies are equal to the relative Stokes parameters $p_{x}, p_{y}$. Due to the good agreement with the linear relations, it could be claimed that these parameters also remain unchanging for each period. Moreover, the average polarization degree $p$ could be found for the different events: $21 \%, 29 \%$, and 9\% . The striking deviations presumably represent the occurrence of rapid polarization state variations that conform well to the theoretical models. It should be mentioned that extrinsic polarization makes a contribution to the total value as well. So, the polarization carried by ISM and AGN components (e.g., accretion disk or dust torus) should be also taken into account. The degree of constant polarization could be 
calculated with system (4). According to the observation process, the value $I^{c}$ is equal to $F_{R}^{c}$; the last value could be found by the flux-flux diagrams. Therefore, the permanent contribution to the polarized flux does not exceed several percent. Additionally, the instrumental polarization is not greater than $2 \%$. These values are negligible compared with variable polarization degree-up to $40 \%$ at outbursts. In summary, the interval of the variable source polarization degree changes is approximately $0-40 \%$; the polarization angle also changes within all possible values, and it is essential to note that evident correlation has not yet been revealed.

Presently, the work is still in progress. To clarify the object behaviour, we are going to find the coefficients of correlation between blazar intensity and its polarization. The time lag between polarized and unpolarized fluxes observation would reveal the polarization origin-inner of extrinsic (e.g., the reflection on the dust torus) — and according to that the conclusions regarding polarization nature could be drawn.

Acknowledgments: The author expresses gratitude to the scientific supervisor Prof. V. A. Hagen-Thorn and the members of Observational Astrophysics laboratory of SPbSU.

Conflicts of Interest: The authors declare no conflict of interest.

\section{References}

1. Blandford, R.D.; Rees, M.J. Extended and Compact Extragalactic Radio Sources: Interpretation and Theory. Phys. Scr. 1978, 31, 265-274.

2. Spinrad, H.; Smith, H.E. AO 0235+164-A Red BL Lacertae Object. Astrophys. J. 1975, 201, 275-276.

3. Stikel, M.; Fried, J.W.; Kuehr, H. The Complete Sample of 1 Jy BL Lac Objects. II. Observational data. Astron. Astrophys. Suppl. Ser. 1993, 98, 393-442.

4. Raiteri, C.M.; Villata, M.; Aller, H.D.; Aller, M.F.; Heidt, J.; Kurtanidze, O.M.; Lanteri, L.; Maesano, M.; Massaro, E.; Montagni, F.; et al. Optical and Radio Variability of the BL Lacertae Object AO 0235+16: A Possible 5-6 Year Periodicity. Astron. Astrophys. 2001, 377, 396-412.

5. Romero, G.E.; Combi, J.A.; Benaglia, P.; Azcarate, I.N.; Cersosimo, J.C.; Wilkes, L.M. Search for intraday radio variability in EGRET blazars. Astron. Astrophys. 1997, 326, 77-86.

6. Rabbette, M.; McBreen, B.; Steel, S.; Smith, N. Rapid optical variability in the BL Lacertae object AO $0235+164$. Astron. Astrophys. 1996, 310, 1-7.

7. Hartman, R.C.; Bertsch, D.L.; Bloom, S.D.; Chen, A.W.; Deines-Jones, P.; Esposito, J.A.; Fichtel, C.E.; Friedlander, D.P.; Hunter, S.D.; McDonald, L.M.; et al. The Third EGRET Catalog of High-Energy Gamma-Ray Sources. Astron. Astrophys. Suppl. Ser. 1999, 123, 79-202.

8. Gabuzda, D.C.; Pushkarev, A.B.; Cawthorne, T.V. The $\lambda=6 \mathrm{~cm}$ VLBI polarization structure of nine BL Lacertae objects. Mon. Not. R. Astron. Soc. 1999, 307, 725-736.

9. Hagen-Thorn, V.A.; Marchenko, S.G. Photometry and Polarimetry of Active Galactic Nuclei. Balt. Astron. 1999, 8, 575-592.

10. Hagen-Thorn, V.A.; Larionov, V.M.; Jorstad, S.G.; Arkharov, A.A.; Hagen-Thorn, E.I.; Efimova, N.V.; Larionova, L.V.; Marscher, A.P. The Outburst of the Blazar AO 0235+164 in 2006 December: Shock-in-Jet Interpretation. Astrophys. J. 2008, 672, 40-47.

11. Efimova, N.V. Color and Polarization Variability of Blazars. Ph.D. Dissertation. Pulkovo Astronomical Observatory of Russian Academy of Science, Saint Petersburg, Russia, 2009; p. 157.

12. Choloniewski, J. The Shape and Variability of the Nonthermal Component of the Optical Spectra of Active Galaxies. Acta Astron. 1981, 17, 295-311.

13. Linnik, Y.V. Method of Least Squares and Principles of the Theory of Observations; Johnson, N.L., Ed.; Pergamon Press: Oxford, UK, 1961; p. 360. 